\title{
AÇõES DE VIGILÂNCIA EM SAÚdE NO CURSO DA PANDEMIA DE COVID-19, EM SOBRAL-CE
}

\author{
HEALTH SURVEILLANCE ACTIONS DURING COVID-19 PANDEMICS IN SOBRAL-CE \\ ACCIONES DE VIGILANCIA EN SALUd EN EL CURSO DE LA PANDEMIA DE LA COVID-19 EN SOBRAL-CE
}

Francisca Leite Mendonça Escócio 1

Francisco Valdicélio Ferreira ${ }^{2}$

Sandra Maria Carneiro Flôr ${ }^{3}$

Amanda Albuquerque Rocha ${ }^{4}$

Suely Torquato Ribeiro ${ }^{5}$

Marcelo Vieira da Silva 6

Palavras-chave:

Vigilância em Saúde Pública; Gestão em Saúde; Pandemias; Infecções por coronavírus.

Keywords:

Public Health Surveillance; Health Management; Pandemics; Coronavirus Infections.

Palabras clave: Vigilancia en Salud Pública; Gestión en Salud; Pandemias; Infecciones por coronavirus.

Submetido: $17 / 11 / 20$

Aprovado: $10 / 12 / 20$

Autor(a) para Correspondência: Francisca Leite Mendonça Escócio Av. Deputado João Frederico Gomes, 618 - Junco Sobral - CE CEP. 62.030-262 E-mail: francisca.escocio@sobral. ce.gov.br.

\section{RESUMO}

A crise sanitária provocada pela pandemia de Covid-19 evidenciou as desigualdades sociais no âmbito da saúde em geral e realçou a relevância da Vigilância em Saúde em medidas de controle da disseminação de doenças, requerendo a redefinição das estratégias planejadas e a incorporação de novas ações. Este artigo objetiva descrever as ações realizadas pelas Células que compõem a Coordenadoria de Vigilância em Saúde da Secretaria Municipal de Saúde de Sobral, Ceará, para o enfrentamento da Covid-19. Trata-se de um relato de experiência com a descrição analítica das ações desenvolvidas pelas gerências das Células da Vigilância Epidemiológica, da Unidade de Vigilância de Zoonoses, da Vigilância Sanitária, da Vigilância em Saúde Ambiental, da Vigilância Alimentar e Nutricional e do Centro de Referência em Saúde do Trabalhador. As ações descritas conferem maior visibilidade à relevância das várias faces da vigilância em saúde diante da gestão, do setor regulado e da população. Todas as ações e respostas ressignificaram a gestão local, propondo uma articulação de políticas intersetoriais voltadas à melhoria da qualidade de vida das pessoas. Essa vivência mostrou a importância do trabalho colaborativo, indo além do fazer específico de cada Célula, fortalecendo as intra e intersetorialidades, em consonância com a Lei n. ${ }^{\circ}$ 8.080/90. Conclui-se que essa experiência evidencia a importância do trabalho e fortalecimento da Coordenadoria de Vigilância em Saúde da Secretaria Municipal de Saúde de Sobral-CE, centrada nas abordagens humana e comunitária.

\footnotetext{
1. Enfermeira, Coordenadoria de Vigilância em Saúde da secretaria da saúde de Sobral-CE (2017-2020), Brasil. E-mail: francisca.escocio@sobral.ce.gov.br. 0RCID: https://orcid.org/0000-0002-2666-4791

2. Nutricionista, Gerente da Célula de Vigilância Alimentar e Nutricional da secretaria da saúde de Sobral-CE, Brasil. E-mail: valdicelioferreira@sobral.ce.gov.br. ORCID: https://orcid.org/0000-0002-6347-2844

3. Enfermeira, Gerente da Célula de Vigilância Epidemiológica da secretaria da saúde de Sobral-CE (2017-2020), Brasil. E-mail: vigepsobral@gmail.com.0RCID: http://orcid.org/0000-0003-2266-9699

4. Médica Veterinária, Gerente da Célula de Unidade de Vigilância em Zoonoses da secretaria da saúde de SobralCE (2017-2020),Brasil.E-mail: amandarocha@sobral.ce.gov.br. 0RCID: https://orcid.org/0000-0003-0835-1182 5. Tecnóloga em Alimentos, Gerente da Célula de Vigilância em Saúde Ambiental da secretaria da saúde de SobralCE, Brasil. E-mail: suely.ribeiro@sobral.ce.gov.br. ORCID: htpps://orcid.orq/0000-0002-6384-3836

6. Enfermeiro da Célula do Centro de Referência em Saúde Trabalhador (CEREST) da secretaria da saúde de SobralCE, Brasil. E-mail: marcelovs23@hotmail.com. ORCID: htpps://orcid.org/0000-0002-6554-8312
} 


\section{ABSTRACT}

The health crisis caused by the Covid-19 pandemics highlighted social inequalities in the field of health in general, and highlighted the relevance of health surveillance in measures to control the spread of diseases, requiring the redefinition of planned strategies and the incorporation of new actions. This article aims to describe the actions taken by the Cells that constitute the Health Surveillance Coordination of the Municipal Department of Health in Sobral, Ceará, to face the Covid-19 pandemics. This is an experience report with an analytical description of the actions developed by the managements of the Epidemiological Surveillance Cells, the Zoonosis Surveillance Unit, the Health Surveillance, the Environmental Health Surveillance, the Food and Nutrition Surveillance and the Reference Center in Occupational Health. The actions described provide greater visibility to the relevance of the various aspects of health surveillance in the face of management, the regulated sector and the population. All actions and responses gave new meaning to local management, proposing an articulation of intersectoral policies aimed at improving people's quality of life. This experience showed the importance of collaborative work, going beyond the specific actions of each Cell, strengthening the intra and intersectoral aspects in line with Law No. 8,080/90. We conclude that this experience highlights the importance of work and strengthening of the Coordination of Health Surveillance of the Municipal Department of Health in Sobral-CE, focused on human and community approach.

\section{RESUMEN}

La crisis sanitaria desencadenada por la pandemia de la Covid-19 evidenció las desigualdades sociales en el ámbito de la salud en general y resaltó la relevancia de la vigilancia en salud en medidas de control de la diseminación de enfermedades, solicitando el restablecimiento de las estrategias planeadas y la incorporación de nuevas acciones. Este artículo tiene como objetivo describir las acciones realizadas por las Células que componen la Coordinación de Vigilancia en Salud de la Covid-19. Se trata de un relato de experiencia con descripción analitica de las acciones desarrolladas por las gerencias de las Células de Vigilancia Epidemiológica, de la Unidad de Vigilancia de Zoonosis, de la Vigilancia Sanitaria, de la Vigilancia en Salud Ambiental, de la Vigilancia Alimentar y Nutricional y del Centro de Referencia en Salud del Trabajador. Las acciones descriptas confieren mayor visibilidad de la relevancia de las varias frentes de la vigilancia en salud delante de la gestión, del sector regulado y de la población. Todas las acciones y respuestas resignificaron la gestión local, proponiendo una articulación de políticas intersectoriales vueltas a la mejoría de la cualidad de vida de las personas. Esa vivencia ha mostrado la importancia del trabajo colaborativo, yendo más allá de lo que hace cada Célula, fortaleciendo la intra e intersectorialidad en consonancia con la Ley N. ${ }^{\circ}$ 8.080/90. Se concluye que esa experiencia evidencia la importancia del trabajo y el fortalecimiento de la Coordinación de Vigilancia en Salud de la Secretaria Municipal de Saúde de Sobral-CE, centrada en el enfoque humano y comunitario.

\section{INTRODUÇÃ 0}

Em dezembro de 2019 foi detectado, em Wuhan, na China, o primeiro caso de Severe Acute Respiratory Syndrome Coronavirus 2 (SARS-CoV-2). Em janeiro de 2020, a Organização Mundial da Saúde (OMS) declarou se tratar de problema com impacto internacional ${ }^{1}$. Neste mesmo mês, o Ministério da Saúde (MS) decretou que casos suspeitos de pneumonia de origem indeterminada deveriam ser notificados em até 24 horas ao Centro de Informações Estratégicas de Vigilância em Saúde (CIEVS). 0 primeiro caso da América Latina ocorreu no Brasil, em 25 de fevereiro de 2020, em São Paulo, cidade mais populosa da América do Sul ${ }^{2}$. Tratava-se de um paciente do sexo masculino, 61 anos, que havia viajado em fevereiro à Lombardia, Itália, onde estava ocorrendo surto significativo ${ }^{3}$.

Em março, no Brasil, existiam 488 casos suspeitos, dois confirmados e 240 descartados. Com isso, imediatamente a Secretaria de Vigilância em Saúde do Ministério da Saúde (SVS/MS) desenvolveu estratégias para a detecção precoce e ativação dos Pontos Focais Nacionais do Regulamento Sanitário Internacional da OMS (PFM-RSI/OMS) ${ }^{4,5}$. Após dois meses do primeiro caso, a transmissão comunitária foi detectada, considerando ocorrências de casos sem vínculo com caso confirmado ou viagem internacional.

Segundo a Nota Técnica GVIMS/GGTES/ANVISA n. ${ }^{\circ}$ 04/20206, de um a quatro dias antes do início dos sintomas da Covid-19 identifica-se transmissão, 
mesmo que o vírus esteja em período de incubação. Portanto, a transmissão pode ocorrer, devendo ser realizados o monitoramento e planejamento sobre os contatos anteriores ao início dos sintomas ${ }^{7}$. A detecção precoce permite monitoramento e controle, visto que não é possível realizar bloqueio por meio de vacinas, sendo o distanciamento físico a principal medida no controle da transmissibilidade.

Nesse contexto, a Vigilância em Saúde é essencial para subsidiar os gestores na tomada de decisão e adoção de estratégias efetivas. Em Sobral, Ceará, o SARS-CoV-2 apresentou alta transmissibilidade. 0 primeiro caso suspeito ocorreu em janeiro, sendo descartado posteriormente. Ocorrência que motivou a elaboração de um plano de contingência com a participação de todo o núcleo gestor da Secretaria da Saúde, sob a articulação da Coordenadoria de Políticas e Planejamento de Atenção à Saúde.

Sobral se organizou por meio da institucionalização de um Comitê de Operação em Saúde Pública de Sobral (COESP-S), com a integração das secretarias municipais, universidades, indústrias, hospitais públicos e privados, a fim de articular estratégias inter e intrasetoriais frente à pandemia. Considerando que a análise de dados epidemiológicos é fundamental para dar subsídio à gestão em saúde, a Secretaria da Saúde instituiu um Comitê de Crise enquanto dispositivo de processamento de dados, planejamento de ações e avaliação, visando à detecção precoce da doença, monitoramento e adequada assistência aos casos e seus contatos.

Em 13 de março de 2020, a Prefeitura Municipal emitiu o Decreto $n .^{\circ} 2.369 / 2020^{8}$, que trouxe em seu texto medidas de contingenciamento da pandemia. Em 31 de maio, o Decreto n. ${ }^{\circ} 2.437$ instituiu o isolamento social rígido como medida ${ }^{9}$.

Em face dessa situação de urgência sanitária, a Coordenadoria de Vigilância em Saúde se articulou com as células que a compõem: Célula do Centro de Referência em Saúde do Trabalhador (CEREST), Célula da Vigilância Epidemiológica, Célula da Unidade de Vigilância de Zoonoses, Célula de Vigilância Sanitária, Célula de Vigilância em Saúde Ambiental e Célula da Vigilância Alimentar e Nutricional para o desenvolvimento de estratégias voltadas ao enfrentamento do SARS-CoV- 2, sem que houvesse descontinuidade das ações previstas na Programação Anual de Saúde.

Este estudo tem o objetivo de descrever as ações realizadas pelas Células que compõem a Coordenadoria de Vigilância em Saúde da Secretaria

\section{"A Vigilância em Saúde \\ estrutura a sua atuação \\ por meio de processo \\ contínuo e sistemático \\ de coleta, consolidação, \\ análise e disseminação de dados"}

de Saúde de Sobral, Ceará, no enfrentamento da pandemia de Covid-19.

\section{METODOLOGIA}

Relato de experiência desenvolvido pela equipe gestora da Coordenadoria da Vigilância em Saúde, que atuou no período de março a novembro de 2020 no enfrentamento da pandemia de Covid-19 em Sobral, município situado na região Noroeste do Ceará, localizado a 235 quilômetros da capital, Fortaleza.

A Vigilância em Saúde estrutura a sua atuação por meio de processo contínuo e sistemático de coleta, consolidação, análise e disseminação de dados relacionados a eventos no contexto da saúde, visando ao planejamento e à implementação de medidas de saúde pública para a proteção da saúde da população, prevenção e controle de riscos, agravos e doenças ${ }^{10,11}$. A Coordenadoria de Vigilância em Saúde de Sobral constitui uma das 10 coordenações da Secretaria da Saúde, representando, num esquema hierárquico de gestão, o primeiro escalão desta secretaria. É composta por sete Células e um núcleo de fiscalização que setorialmente desenvolvem processos específicos à área de atuação, mas que se mantêm organicamente articulados e orientados pelo mesmo fim: promover a vigilância à saúde de todos os cidadãos circunscritos ao seu território de atuação. Para tanto, organiza-se em: Vigilância Sanitária (VS), Vigilância Epidemiológica (VE), Vigilância Ambiental em Saúde (VAS), Vigilância em Saúde do Trabalhador (VST), Vigilância de Zoonoses (VZ) e Vigilância Alimentar e Nutricional (VAN). Estas duas últimas foram instituídas pelo município, ampliando o que já é preconizado.

Os relatos deste artigo foram sistematizados pelos profissionais que vivenciaram as experiências e serão expostos em tópicos que se referem a cada gerência da coordenação de vigilância à saúde, com o intento de conferir mais clareza ao fazer de 
cada uma, expondo-as à análise crítica, a partir de diálogo com a literatura apropriada.

\section{RESULTADOS E DISCUSSÃO}

Os resultados desta experiência serão estruturados com base na atuação das Células vinculadas à Coordenadoria de Vigilância em Saúde. Registrase o fazer específico de cada Célula e as ações especificamente deflagradas e/ou intensificadas no curso da pandemia de Covid-19. A partir disso, tecese análise referenciada por autores com produção de literatura afim.

\section{Célula de Vigilância Epidemiológica}

Entende-se como Vigilância Epidemiológica um conjunto de ações que proporcionam o conhecimento e a detecção de mudanças nos fatores determinantes e condicionantes da saúde individual e coletiva, com a finalidade de recomendar e adotar as medidas de prevenção e controle das doenças ou agravos ${ }^{12}$.

A Vigilância Epidemiológica de infecção pelo SARS-CoV-2 foi construída à medida que a OMS consolidava informações e novas evidências técnicas e científicas eram publicadas por meio de boletins, notas técnicas e manuais ${ }^{13}$. Dessa forma, foi elaborado um plano com recomendações sobre: notificação, registro de investigação, manejo e adoção de medidas preventivas. Essa iniciativa foi significativa para o processo de análise e tomada de decisão no enfrentamento da pandemia. Em parceria com uma equipe interprofissional, composta por: enfermeiro, fisioterapeuta, nutricionista e profissional de educação física, vinculada às residências multiprofissionais em saúde e ao núcleo de apoio multiprofissional, a célula de Vigilância Epidemiológica do município de Sobral traçou estratégias para o enfrentamento e controle da Covid-19.

A primeira intervenção para o controle e prevenção da doença ocorreu na segunda quinzena de março de 2020. Tratou-se de reunião com os gerentes dos Centros de Saúde da Família (CSF), coordenadores de enfermagem das unidades hospitalares e profissionais da gestão da saúde do município. Na ocasião, Sobral registrava dois casos suspeitos da doença. Foram apresentados os instrumentos de notificação, de acordo com a definição de caso suspeito, fluxograma para atendimento e manejo dos casos, além das medidas preventivas.

\section{"A Célula de epidemiologia realizou monitoramento e avaliação de todas as Declarações de Óbito (DO) que faziam menção à Covid-19"}

A ficha de notificação da Síndrome Respiratória Aguda Grave (SRAG), criada pelo MS $^{14}$, foi implantada nos hospitais para registros no Sistema de Informação da Vigilância Epidemiológica da Gripe (SIVEP - GRIPE). Por intermédio das notificações ambulatoriais, hospitalares, de laboratórios públicos e privados, verificou-se que cresciam os números de casos, repercutindo no acréscimo de hospitalizações, tendo, alguns, como desfecho, o óbito.

No que concerne à vigilância de óbitos por Covid-19, a Célula de epidemiologia realizou monitoramento e avaliação de todas as Declarações de Óbito (D0) que faziam menção à Covid-19 como causa. Também foi realizada busca ativa de D.0 nos estabelecimentos de saúde, cartórios de registro civil e Instituto de Medicina Legal (IML). Esse fluxo de busca está previsto em portaria do MS, considerando que o registro civil do óbito pode ser realizado no município de ocorrência ou de residência do falecido.

A avaliação das D.0 é realizada pela equipe da Vigilância Epidemiológica para a identificação das causas informadas na declaração pelos médicos atestantes, sendo, posteriormente, codificada e digitada no Sistema de Informação sobre Mortalidade (SIM). Também é realizado o cruzamento dos óbitos com o SIVEP - GRIPE e encerramento do caso no sistema. Em seguida, é alimentada a planilha de monitoramento de óbitos por Covid-19 e enviada para a Superintendência da região Norte, a qual alimenta, em tempo real, o Integra SUS, para o encerramento dos casos de acordo com a classificação final no e-SUS.

Outra ação importante foi a implantação da ficha de notificação de acidente de trabalho grave para os casos de adoecimento de profissionais de saúde, por Covid-19. Em parceria com o CEREST, profissionais dos hospitais: Dr. Estevam Ponte, Hospital Dr. Alves (de campanha) e Hospital Santa Casa de Misericórdia de Sobral foram capacitados.

Para a descentralização do e-SUS e consequente notificação dos casos pela Atenção Primária à 
Saúde (APS), foram capacitados 60 profissionais dentistas e enfermeiros com o intuito de rastrear e monitorar os contatos, preferencialmente nas primeiras 48 horas após a notificação do caso, para interromper as cadeias de transmissão e, consequentemente, promover a diminuição de novos casos $^{3}$. Quanto mais descentralizada a notificação e o registro, mais oportuna será a informação dos dados epidemiológicos.

Até o dia 20 de novembro de 2020, somam-se 12.191 casos confirmados; destes, 12.053 foram recuperados e 318 faleceram. Até essa data, foram testadas 29.038 pessoas, correspondendo a 13,9\% da população de Sobral ${ }^{15}$. Registra-se um decréscimo significativo no número de casos novos e de internações, o que não desobriga a manutenção de todas as medidas relatadas e permanente ação da Vigilância Epidemiológica.

\section{Célula de Vigilância de Zoonoses}

Unidades de Vigilância de Zoonoses são estruturas físicas e técnicas responsáveis pela execução de parte ou da totalidade das atividades, ações e estratégias referentes à vigilância, à prevenção e ao controle de zoonoses e de acidentes causados por animais peçonhentos e venenosos, de relevância para a saúde pública ${ }^{16}$. Conforme o Manual de Vigilância, Prevenção e Controle de Zoonoses ${ }^{17}$, suas ações se pautam em atuar e intervir, direta ou indiretamente, sobre as populações de animais-alvo, de modo a refletir em benefício direto (redução ou eliminação, quando possivel, do risco iminente de transmissão de zoonose) à saúde da população humana.

Em Sobral, a referida unidade é responsável pela execução dos Programas de Vigilância e Controle das doenças zoonóticas: Arboviroses, Doença de Chagas, Raiva e Leishmanioses, Programa de Vigilância e Controle do Tracoma e Monitoramento de Animais Peçonhentos. As ações efetuadas nos territórios são realizadas pelos Agentes de Combate às Endemias $(A C E)$, em visitas domiciliares.

Em virtude da pandemia de Covid-19, em 26 de março de 2020 o MS emitiu a Nota Informativa n. ${ }^{0}$ 08/2020-CGARB/DEIDT/SVS/MS ${ }^{18}$, com recomendações aos ACE para a adequação das ações de vigilância e controle de zoonoses frente à nova situação epidemiológica. Na Nota, ficam expressas as orientações sobre o afastamento de servidores sintomáticos gripais, comunicação ao setor competente de casos sintomáticos identificados

\section{"Até o dia 20 de novembro de 2020, somam-se 12.191 casos confirmados; destes, 12.053 foram recuperados e 318 faleceram"}

nas visitas domiciliares e não realização de visitas em casas com moradores acima de 60 anos. As visitas domiciliares foram redimensionadas, com a inspeção passando a ser realizada exclusivamente no peridomicílio; recomendação aos moradores sobre a importância da adoção de medidas preventivas, sobretudo no que se refere a criatórios do mosquito Aedes aegypti; realização de bloqueio da transmissão em áreas com intensa circulação de vírus (dengue, chikungunya e/ou zika); distanciamento social; e assepsia adequada das mãos.

Considerando o aumento do índice de infestação predial pelo mosquito $A$. aegypti em decorrência da chegada do período chuvoso, bem como o aumento das notificações de dengue no período de março a maio, foram realizadas intensas medidas de controle vetorial com a finalidade de prevenir a epidemia dessa arbovirose. Enfatiza-se o Programa de Vigilância e Controle das Arboviroses pela presença dessas doenças, especialmente a dengue, no Ceará, sendo esta a arbovirose urbana de maior relevância nas Américas e que tem se constituído como problema de saúde pública mundial ${ }^{19}$.

A partir da publicação da Nota Informativa $08 / 2020^{18}$, as atividades dos ACE foram direcionadas para a inspeção em pontos estratégicos de proliferação do mosquito $A$. aegypti, além do monitoramento de terrenos baldios e imóveis abandonados. 0s servidores receberam equipamentos de proteção individual (EPIs) como máscaras $\mathrm{N} 95$ e protetores faciais plásticos. Também foi fornecido álcool $70 \%$ para a higienização das mãos. Nos territórios mais críticos (com índice de infestação mais elevado e grande quantidade de notificações), foram intensificadas as vistorias e realizado o bloqueio vetorial através de controle químico com o carro de Ultrabaixo Volume (UBV Pesada) e bombas costais. Para o acompanhamento do resultado das ações, semanalmente os dados eram georreferenciados para a análise espacial por meio de mapas com os 
índices de infestação predial por território. Essa ferramenta, em análise conjunta com os dados extraídos do Sistema de Informação de Agravos de Notificação (SINAN), fornecidos pela gerência de Vigilância Epidemiológica, permitiu a observação em tempo oportuno da dinâmica de distribuição do mosquito e de casos notificados para a tomada de decisões assertivas ${ }^{20}$.

Destacamos, aqui, a importância das ações intersetoriais durante a pandemia e o reconhecimento de cada setor no esforço de combate às arboviroses. Em março de 2020 foi criado o Comitê de Monitoramento da Dengue, com a participação da Coordenação de Vigilância em Saúde, Coordenação de Políticas e Planejamento de Atenção à Saúde, Coordenação de Atenção Primária, Escola de Saúde Pública Visconde de Saboia (ESP-VS) e Secretaria da Saúde. Elaborouse um plano de mídia para a divulgação das ações de prevenção nas comunidades, incluindo rádios e materiais para a circulação em redes sociais. Salientamos a parceria nas ações de comunicação da Escola de Saúde Pública Visconde de Saboia, com a gravação de vídeos com teatro de bonecos realizados pelo Grupo de Trabalho de Arte e Educação Popular em Saúde, bem como da Secretaria dos Direitos Humanos e Assistência Social (SEDHAS), com o projeto Bike Sonora e Vetincionário, com a circulação de spots e publicação de vídeos sobre arboviroses, gravados por pessoas da comunidade, em linguagem acessível. Por fim, enfatizamos as ações realizadas em parceria com a Secretaria de Serviços Públicos na limpeza de terrenos baldios, em especial naqueles em bairros críticos, mapeados pelos ACE, e a identificação, com posterior notificação, de proprietários de imóveis abandonados ou terrenos murados com acúmulo de entulhos e lixo.

\section{Célula de Vigilância Sanitária}

A expressão "vigilância sanitária" é própria do Brasil, mas ações de regulação e vigilância sanitária são práticas universais. Em todas as épocas houve intervenções do poder de autoridade sobre as práticas de cura, os medicamentos, os alimentos, a água e o ambiente ${ }^{21}$.

Vigilância Sanitária constitui um espaço institucional, determinado historicamente e pertence à área da Saúde Coletiva enquanto campo de conhecimento e âmbito de práticas. Compete à VS o desenvolvimento de ações estratégicas no sistema de saúde, regulação sanitária das atividades

\section{"Destacamos, aqui, a importância das ações intersetoriais durante a pandemia e o reconhecimento de cada setor no esforço de combate às arboviroses"}

relacionadas ao ciclo de produção/consumo de bens e serviços de interesse da saúde, das esferas privada e pública. Sua dinâmica se vincula ao desenvolvimento científico e tecnológico e processos políticos que perpassam o Estado, o mercado e as sociedades, nos âmbitos interno e internacional ${ }^{22,23}$.

A Vigilância Sanitária tem o compromisso de garantir o cumprimento dos decretos municipais e estaduais, orientar e estabelecer parâmetros técnicos aos profissionais e estabelecimentos voltados aos diversos produtos e serviços de interesse à saúde, bem como serviços de alimentação e produtos alimentícios ${ }^{24}$. Alinhada ao Plano Municipal de Contingência para a Covid-19, a Vigilância Sanitária tem exercido papel fundamental, com intensificação das responsabilidades mencionadas e focalizando na fiscalização e orientação dos estabelecimentos em funcionamento, averiguando as condições sanitárias.

Sumariamente, a Vigilância Sanitária, no curso da pandemia de Covid-19, agiu por meio de: (I) Orientação para o setor regulado, trabalhadores e população para a adoção de medidas de prevenção e controle da infecção pelo SARS-CoV-2; (II) Orientação quanto às medidas de prevenção e controle de infecção para casos suspeitos ou confirmados nos serviços de interesse sanitário; (III) Fiscalização in loco do cumprimento da legislação pelos estabelecimentos submetidos ao regime de Vigilância Sanitária; (IV) Controle e a fiscalização de substâncias e produtos de interesse para a saúde; (V) Assessoramento no processo de normatização da gestão relacionada ao enfrentamento da Covid-19; (VI) Recebimento e atendimento às denúncias; (VII) Monitoramento de casos suspeitos e confirmados de Covid-19 que estavam descumprindo orientações de isolamento e/ ou quarentena; (VIII) Apoio às barreiras sanitárias.

0 enfrentamento da pandemia apontou fragilidades do sistema de saúde, repercutindo na vigilância. Barreiras históricas como subfinanciamento de décadas e ações para a desestruturação do Sistema 
Único de Saúde (SUS) limitam sua capacidade de responder aos problemas e necessidades colocados pela pandemia ${ }^{25}$. Contudo, no curso da pandemia, ao assumir novas demandas, a VS tem a oportunidade de conferir maior visibilidade às suas ações diante da gestão, do setor regulado e da população, ressignificando práticas e propondo articulação intersetorial na busca pela melhoria da qualidade de vida das pessoas.

\section{Célula do Centro de Referência em Saúde do Trabalhador}

A Saúde do Trabalhador compreende a produção de conhecimento, a utilização de tecnologias e práticas de saúde, seja no plano técnico ou político, visando à promoção da saúde e à prevenção de doenças, sejam de origem ocupacional ou relacionadas a o trabalho ${ }^{24}$. No Brasil, a Saúde do Trabalhador, entendida como campo de práticas apoiadas no modelo de Saúde Pública, disseminou-se com o Movimento da Reforma Sanitária e se desenvolveu mais amplamente a partir da promulgação da Constituição de 1988 e a implementação do Sistema Único de Saúde ${ }^{26}$.

No contexto do SUS é que surge a Rede Nacional de Atenção Integral à Saúde do Trabalhador (Renast), que possui o papel de unir e criar interações entre os serviços de saúde do trabalhador, a rede de saúde do Brasil e demais segmentos da sociedade responsáveis e engajados na questão da saúde dos trabalhadores. Nesta, os Centros de Referência em Saúde do Trabalhador (Cerest) representam serviços articuladores da rede e de retaguarda do SUS, conforme a Portaria n. ${ }^{0} 1.679^{27}$.

Diante da grave crise sanitária com fortes afetações sobre os trabalhadores, o Cerest de Sobral vem desenvolvendo ações no sentido de identificar riscos nos ambientes de trabalho, compartilhar protocolos de proteção individual e coletiva aos trabalhadores, realizar notificações de trabalhadores contaminados pelo SARS-CoV-2 em seus ambientes de trabalho, monitoramento e capacitação para as equipes das Unidades Sentinela, além de outras ações voltadas para a Vigilância Epidemiológica em Saúde do Trabalhador em toda a área de abrangência. Nesse sentido, destacam-se as atividades realizadas pelo Cerest/Sobral no período de 23 de março a 30 de outubro de 2020, em vigência da pandemia de Covid-19:

- 301 inspeções sanitárias em Saúde do Trabalhador em parceria com as Vigilâncias

\section{"Tanto o exercício das atividades laborais quanto as condições de trabalho são fontes potenciais de exposição ao vírus"}

Sanitária, Ambiental e Nutricional, com o objetivo de identificar riscos e verificar medidas de proteção individual e coletiva entre os trabalhadores;

- 908 notificações de Acidente de Trabalho Grave de trabalhadores da saúde e segurança contaminados pelo novo coronavírus, atendendo à Recomendação Técnica do Estado do Ceará, de 15 de maio de 2020, que orienta a notificação compulsória nessas situações;

- $\quad 52$ monitoramentos em Unidades Sentinela de referência em Doenças e Agravos relacionados ao Trabalho;

- 02 encontros virtuais com representantes dos municípios da área de abrangência acerca das ações de proteção contra a pandemia, voltadas para os trabalhadores.

No decorrer das ações realizadas, ficou evidente que tanto o exercício das atividades laborais quanto as condições de trabalho são fontes potenciais de exposição ao vírus. Por sua vez, esse lócus trabalho - é território de disseminação da doença. Portanto, é fundamental entender de que maneira as atividades e condições de trabalho podem contribuir para a disseminação e estabelecimento de estratégias de enfrentamento da Covid-19. Assim, para assegurar condições laborais que propiciem a redução na transmissão do vírus, medidas organizacionais necessitam ser discutidas no âmbito de cada atividade de trabalho e a práxis da Saúde do Trabalhador deve ser considerada no rol das medidas e ações de Saúde Pública voltadas ao controle da pandemia ${ }^{27}$.

\section{Célula de Vigilância em Saúde Ambiental}

Vigilância em Saúde Ambiental é o campo da saúde pública que reúne conhecimentos, políticas públicas e ações relacionadas à interação entre saúde humana e fatores ambientais, que determinam, condicionam e influenciam a qualidade de vida das pessoas. Essas ações compreendem a articulação dos saberes, 
processos e práticas que foram intensificadas no curso da pandemia de Covid-19, sendo necessários planos de enfrentamento, fazendo-se importante distribuir as responsabilidades e obrigações de cada setor da sociedade brasileira ${ }^{28}$.

Uma das ações intensificadas no período de pandemia foi a vigilância e monitoramento da qualidade da água destinada ao consumo humano, tal intensificação de ações justifica-se pelo fato de a água ser identificada como indicador essencial e, consequentemente, pautada no estudo precursor que avaliou as rotas de transmissão de doenças relacionadas à água, classificando-as em quatro categorias, das quais duas são de especial relevância para a Covid-19, sendo elas: a) risco contido na água, onde a transmissão ocorre por meio da ingestão de um patógeno presente na água, atuando como veículo para agentes infecciosos; b) pautada na água como instrumento de higiene, onde a infecção pode ser evitada a partir do fornecimento de água suficiente para a higiene doméstica e pessoal ${ }^{29}$.

Portanto, a transmissão do vírus pode ser reduzida pela disponibilidade de oferta de água na quantidade e qualidade desejadas, sendo o primeiro nível de intervenção de Vigilância em Saúde Ambiental como importante instrumento para promover a saúde. À medida que a pandemia de Covid-19 se expandia, a importância do acesso à água potável e ao saneamento foi reforçada. A OMS, em suas orientações provisórias de emergência, trouxe como elemento principal a “Água, saneamento, higiene e gerenciamento de resíduos para a Covid-19"29,30.

Várias medidas foram intensificadas para melhorar a segurança da água no município de Sobral, começando com a inspeções em todas as centrais de tratamentos; monitoramento contínuo dos veículos transportadores de água potável; monitoramento dos parâmetros indicadores de qualidade de água, por meio de coletas amostrais semanais e análises em campo, com a finalidade de garantir que seja levada a todos os usuários água potável; bem como a vigilância dos resíduos gerados em unidades de saúde, a fim de prevenir o contágio, enquadrado na classe de risco 3 (alto risco de propagação de pessoa para pessoa e moderado risco de disseminação no meio ambiente).

Desde as primeiras publicações sobre 0 SARS-CoV-2, suspeitava-se que o vírus pudesse ser transmitido pelas vias respiratórias, como perdigotos, espirros e tosses. Contudo, pesquisas recentes também analisaram formas ativas em

\section{"Uma das ações intensificadas no período de pandemia foi a vigilância e monitoramento da qualidade da água destinada ao consumo humano"}

superfícies e objetos contaminados e em efluente ${ }^{31}$. Esses resultados indicam que os resíduos podem ser veículos de transmissão do novo coronavírus e representam risco à população e aos operadores que atuam na coleta, tratamento e destinação adequada. Portanto, outra atuação dessa vigilância foi a gestão e o monitoramento dos Resíduos de Serviço de Saúde (RSS) gerados nas unidades de saúde, garantindo que o tratamento e disposição não fossem interrompidos e que os resíduos infecciosos coletados fossem tratados e descartados com segurança, evitando risco de novas infecções e poluição, assim como medidas protetivas para os funcionários que lidam no gerenciamento e coleta de resíduos.

\section{Célula da Vigilância Alimentar e Nutricional}

0 Sistema de Vigilância Alimentar e Nutricional ${ }^{32}$ foi instituído pelo Ministério da Saúde, pela Portaria n. ${ }^{0} 1.156$, de 31 de agosto de 1990, e está presente na Lei Orgânica da Saúde (Lei n. ${ }^{\circ} 8.080$, de 19 de setembro de 1990), que incluiu a Vigilância Alimentar e Nutricional e a orientação alimentar no campo de atuação do SUS, com registros no Sistema de Vigilância Alimentar e Nutricional (Sisvan). As Portarias n. 79 e $n .^{0} 80$, de 16 de outubro de 1990, estabeleceram estratégias de apoio técnico e operacional para a prática de VAN e a implementação do Sisvan.

A VAN, enquanto componente da Vigilância em Saúde, potencializa as ações de alimentação e nutrição de Atenção Básica ( $A B)$, tendo o papel fundamental de apoiar os gestores e profissionais de saúde no processo de organização e avaliação da atenção nutricional, permitindo que sejam definidas prioridades com base no acompanhamento de indicadores da população assistida. No município de Sobral, a VAN é a gerência atuante nos determinantes da saúde alimentar e nutricional da população, com acompanhamento de programas sociais como - Programa Bolsa Família, Programa Nacional de 
Suplementação de Vitamina A, Programa Nacional de Suplementação de Ferro, Estratégia Amamenta e Alimenta Brasil, Sisvan, NutrisuS e Programa de Alimentação e Nutrição para Necessidades Alimentares Especiais.

No curso da Covid-19, a VAN atuou conforme orientações do Ministério da Saúde, por meio da Nota Técnica n. ${ }^{0} 11 / 2020^{33}$, da Secretaria de Atenção Primária à Saúde, Departamento de Promoção da Saúde e Coordenação-Geral de Promoção da Atividade Física e Ações Intersetoriais, sobre o acompanhamento do Programa Bolsa Família nas condicionalidades da saúde. 0 Bolsa Família é uma política pública que contribui para o combate à pobreza e à desigualdade no Brasil, que desde 2003 tem acompanhado mais de 14 milhões de família. Em Sobral, em média, são 16 mil famílias beneficiárias ${ }^{34}$. No curso da pandemia, - MS flexibilizou o acompanhamento de mulheres e crianças, priorizando o acompanhamento das gestantes.

Os demais programas da Célula da Vigilância Alimentar e Nutricional seguiram seus fluxos com a garantia do seguimento das etiquetas sanitárias. Sobre o Programa Nacional de Suplementação de Vitamina $A^{35}$, cujo objetivo é reduzir e controlar a hipovitaminose $A$, a mortalidade e a morbidade em crianças de seis a 59 meses de idade, os acompanhamentos, no curso da pandemia, foram dirigidos para as crianças que compareceram às unidades de saúde para realizar vacinação ou outro procedimento. Não foi realizada busca ativa e nem suplementação no domicílio durante o pico de contaminação, evitando exposição dos profissionais e das crianças.

Quanto ao Programa Nacional de Suplementação de Ferro ${ }^{36}$, foram raros os atendimentos nas unidades de saúde, no curso da pandemia. As ações previstas no Sisvan-Web se referem a estratégias de educação em saúde relacionadas à alimentação e nutrição, sendo inviável a realização no pico de pandemia. Já a fortificação da alimentação infantil com micronutrientes em pó (NutriSUS) foi interrompida devido ao cancelamento das aulas. 0 Programa Estratégia Amamenta e Alimenta Brasil ${ }^{37}$ também foi interrompido, uma vez que ocorre como grupos. Contudo, o Programa de Assistência Nutricional para Necessidades Alimentares Especiais ficou restrito aos pacientes que apresentaram intercorrências clínicas e que necessitavam de acompanhamento multiprofissional.

Em todo esse contexto de pandemia tivemos

\section{"Tivemos que inovar e adquirir um novo fazer, de forma que gerasse o impacto esperado e não apresentasse risco à prevenção da Covid-19"}

que inovar e adquirir um novo fazer, de forma que gerasse o impacto esperado e não apresentasse risco à prevenção da Covid-19. Desse modo, a equipe da Célula da Vigilância Alimentar e Nutricional apoiou a Vigilância Sanitária em inspeções em ambientes como restaurantes, padarias, mercado público e hotelaria, demonstrando a importância do trabalho colaborativo, indo além do fazer específico de cada Célula, fortalecendo, assim, a intersetorialidade e indo em consonância ao que diz a Lei n. ${ }^{0} 8.080 / 90^{26}$, nos artigos 12 e 13, que enfatizam a intersetorialidade das vigilâncias. Essa experiência demonstrou também a importância da multiprofissionalidade e da promoção da saúde.

\section{CONCLUSÃO}

Essa vivência em Sobral evidenciou a importância e o fortalecimento, no que diz respeito às Células que compõem a Coordenadoria de Vigilância em Saúde, no enfrentamento da Covid-19. Foi abordado neste relato de experiência que a Coordenadoria de Vigilância em Saúde está diretamente relacionada à realização de ações de vigilância, prevenção e controle de doenças transmissíveis, vigilância de fatores de risco e para o desenvolvimento de doenças crônicas não transmissíveis, saúde ambiental e do trabalhador, bem como a análise de situação de saúde da população.

Dessa forma, buscou-se contribuir com a identificação de medidas e desenvolvimento de ações que subsidiaram, no cenário descrito, respostas desenvolvidas pela Coordenação da Vigilância em Saúde, tendo em vista a complexidade das atuações apresentadas, no que se refere ao combate à pandemia causada pela Covid-19, priorizando ações fundamentais para conter a propagação do vírus e da doença. Percebemos, com isso, o fortalecimento dos processos de trabalho colaborativo, ancorados na multiprofissionalidade e intersetorialidade. 


\section{CONTRIBUIÇÃO DOS AUTORES}

Francisca Leite Mendonça Escócio contribuiu com redação, análise e revisão crítica do manuscrito. Francisco Valdicélio Ferreira contribuiu com redação, análise e revisão crítica do manuscrito. Sandra Maria Carneiro Flôr contribuiu com redação, análise e revisão crítica do manuscrito. Amanda Albuquerque Rocha contribuiu com redação, análise e revisão crítica do manuscrito. Suely Torquato Ribeiro contribuiu com redação, análise e revisão crítica do manuscrito. Marcelo Vieira da Silva contribuiu com redação, análise e revisão crítica do manuscrito.

\section{REFERÊNCIAS}

1. Huang C, Wang Y, Li X, Ren L, Zhao J, Hu Y, et al. Clinical features of patients infected with 2019 novel coronavirus in Wuhan, China. Lancet [serial on the internet]. 2020 [cited 2020 Nov 13];395(15):497506. Available from: https://www.thelancet.com/ journals/lancet/article/PIIS0140-6736(20)30183-5/ fulltext

2. Brasil. Boletim Epidemiológico. Infecção humana pelo novo Coronavírus (2019-nCoV). Brasília (DF): Ministério da Saúde; 2020.

3. Rodriguez-Morales $A J$, Katterine $B A D$, Tiwari $R$, Sah R, Rabaan AA, Dhama K. CoVID-19, an Emerging Coronavirus Infection: Current Scenario and Recent Developments - An Overview. J Pure Appl Microbiol [serial on the internet]. 2020 [cited 2020 Nov 8];2020:20200301. Available from: $\quad$ https://search.bvsalud.org/globalliterature-on-novel-coronavirus-2019ncov/resou rce/en/covidwho-143111

4. World Health Organization. Coronavirus disease 2019 (COVID-19) situation reports [document on the internet] 2020 [cited 2020 0ct 20]. Available from: https://www.who.int/docs/default-source/ coronaviruse/situation-reports/20200329-sitrep69-covid-19.pdf?sfursn $=8 \mathrm{~d} 6620 \mathrm{fa} 8$

5. World Health Organization. Coronavirus disease 2019 (COVID-19) situation reports-69. [document on the internet] 2020 [cited 2020 0ct 30]. Available from: https://www.who.int/docs/default-source/ coronaviruse/situation-reports/20200329-sitrep69-covid-19.pdf?sfvrsn $=8 \mathrm{~d} 6620 \mathrm{fa} 8$

6. Agência Nacional de Vigilância Sanitária. Nota técnica n. ${ }^{0}$ 04/2020 - GVIMS/GGTES/ANVISA. Orientações para serviços de saúde: medidas de prevenção e controle que devem ser adotadas durante a assistência aos casos suspeitos ou confirmados de infecção pelo novo coronavírus (SARS-CoV-2). Brasília (DF): Anvisa; 2020.
7. Adhikari SP, Meng $S$, Wu $Y$, Mao $Y$, Ye R, Wang $Q$, et al. Epidemiology, causes, clinical manifestation and diagnosis, prevention and control of coronavirus disease (COVID-19) during the early outbreak period: a scoping review [serial on the internet]. 2020 [cited 2020 Nov 8];9(1):29. Available from: https://www. ncbi.nlm.nih.gov/pmc/articles/PMC7079521/

8. Sobral. Decreto n. ${ }^{0} 2.369$, de 13 de março de 2020 [document on the internet]. Sobral: Prefeitura Municipal de Sobral; 2020 [cited 2020 Nov 10]. Available from: http://www.sobral.ce.gov.br/ diario/public/files/diario/00ebe56060c94df734b04 637524ca2eb.pdf

9. Sobral. Decreto n. ${ }^{0} 2.387$, de 29 de março de 2020 [document on the internet]. Sobral: Prefeitura Municipal de Sobral; 2020 [cited 2020 0ct 30]. Available from: http://www.sobral.ce.gov.br/diario/public/ files/diario/bc88ae180ed744586de2c7978efa0086. pdf.

10. Brasil. Portaria $n^{0} 1.378$, de 9 de julho de 2013. Regulamenta as responsabilidades e define diretrizes para execução e financiamento das ações de Vigilância em Saúde pela União, Estados, Distrito Federal e Municípios, relativos ao Sistema Nacional de Vigilância em Saúde e Sistema Nacional de Vigilância Sanitária. Diário Oficial da União, Brasília, DF, 9 jul. 2013.

11. Conselho Nacional de Secretárias Municipais de Saúde. Vigilância em Saúde: Parte 1. Brasília, (DF); 2011. (Coleção Para Entender a Gestão do SUS, 5).

12. Brasil. Guia de vigilância epidemiológica. Vigilância de Síndromes Respiratórias Agudas COVID-19 [document on the internet]. Brasilia (DF): Ministério da Saúde; 2020 [cited 2020 0ct 27]. Available from: https://portalarquivos. saude.gov.br/images/af gvs coronavirus $6 a g$ o20 ajustes-finais-2.pdf.

13. Brasil. Centro de Operações de Emergências em Saúde Pública. Boletim Epidemiológico. Infecção humana pelo novo Coronavírus (2019-nCoV). Brasília (DF): Ministério da Saúde; 2020.

14. Brasil. Guia de vigilância epidemiológica. Vigilância de Síndromes Respiratórias Agudas COVID-19 [document on the internet]. Brasilia (DF): Ministério da Saúde; 2020 [cited 2020 0ct 27]. Available from: https://portalarquivos. saude.gov.br/images/af gvs coronavirus $6 a g$ o20 ajustes-finais-2.pdf

15. Sobral. Boletim epidemiológico [document on the internet]. Sobral: Secretaria de Saúde; 2020 [cited 2020 0ct 27]. Available from: http://www.sobral. ce.gov.br/informes/principais/boletim-covid-19em-sobral.

16. Brasil. Manual de vigilância, prevenção e controle de zoonoses: normas técnicas e operacionais. Brasília (DF): Ministério da Saúde; 2016. 
17. Brasil. Manual de normas técnicas para estruturas físicas de unidades de vigilância de zoonoses [document on the internet]. Brasília (DF): Ministério da Saúde; 2017 [cited 2020 0ct 27]. Available from: http://bvsms.saude.gov.br/bvs/publicacoes/ normas tecnicas estruturas fisicas unidades vigilancia zoonoses.pdf

18. Brasil. Nota informativa $.^{\circ} 8 / 2020$ - CGARB/ DEIDT/SVS/MS [document on the internet]. Brasília: Ministério da Saúde, Secretaria de Vigilância em Saúde; 2020 [cited 2020 out 30]. Available from: https://coronavirus.ceara.gov.br/wp-content/ uploads/2020/03/covid19 espce Nota-Informativa08-2020-sobre-os-ACES.pdf

19. Brasil. Guia de Vigilância em Saúde. 3. ed. Brasília (DF): Ministério da Saúde; 2019.

20. Brasil. Manual de vigilância, prevenção e controle de zoonoses: normas técnicas e operacionais. Brasília (DF): Ministério da Saúde; 2016.

21. Rosen G. Uma história da Saúde Pública. São Paulo: Hucitec; 1994.

22. Costa EA, Souto AC. Área Temática de Vigilância Sanitária. In: Paim JS, Almeida Filho NA, organizadores. Saúde Coletiva: Teoria e Prática. Rio de Janeiro: Artmed; 2014. p. 327-341.

23. Costa EA. Fundamentos da vigilância sanitária. In: Costa EA, organizadora. Vigilância Sanitária: Temas para Debate. Salvador: EDUFBA; 2009. p. 1136.

24. Gomez CM, Vasconcellos LCF, Machado JMH. Saúde do trabalhador: aspectos históricos, avanços e desafios no Sistema Único de Saúde. Cien Saúde Coletiva [serial on the internet]. 2018 [cited 2020 Nov 09];23(6):1963-70. Available from: http://www.scielo.br/scielo.php?script=sci arttext\&pid $=$ S1413-81232018000601963\&lng $=e n$

25. Brasil. A gestão de riscos e governança na pandemia por covid-19 no brasil: análise dos decretos estaduais no primeiro mês. relatório técnico e sumário executivo. Brasília (DF): Ministério da Saúde, Fundação 0swaldo Cruz; 2020.

26. Brasil. Lei 8.080, de 19 de setembro de 1990. Dispõe sobre as condições para a promoção, proteção e recuperação da saúde, a organização e o funcionamento dos serviços correspondentes e dá outras providências. Brasília (DF): Presidência da República; 1990.

27. Plataforma RENAST Online. Saúde do Trabalhador [document on the internet]. 2020 [cited 2020 Nov 02]. Available from: https://renastonline.ensp. fiocruz.br/temas/saude-trabalhador

28. Abreu LC. Ações integradas e fortalecimento do Sistema Único de Saúde no Brasil em um momento de pandemia. J Hum Growth Dev [serial on the internet]. 2020 [cited 2020 Nov 02];30(1):05-08. Available from: http://pepsic. bvsalud.org/scielo.php?script $=\mathrm{sci}$ arttext \&pid $=S 0104-12822020000100001 \& \operatorname{lng}=p t \& n r m=i s 0$ $\& t \operatorname{lng}=p t$

29. França SAS. A importância do direito à água e ao saneamento para o combate à Covid-19. Paper do NAEA [serial on the internet]. 2020 [cited $2020 \mathrm{Nov}$ 02];29(1). Available from: https://periodicos.ufpa. br/index.php/pnaea/article/view/8769

30. Huang C, Wang Y, Li X, Ren L, Zhao J, Hu Y, et al. Clinical features of patients infected with 2019 novel coronavirus in Wuhan, China. Lancet [serial on the internet]. 2020 [cited 2020 Nov 8];395(15):497506. Available from: https://www.thelancet.com/ journals/lancet/article/PIIS0140-6736(20)30183.5/ fulltext

31. Araújo E, Silva VF. A gestão de resíduos sólidos em época de pandemia do COVID-19. GeoGraphos [serial on the internet]. 2020 [cited 2020 Nov 8];11(129):192-215. Available from: https://web. ua.es/es/revista-geographos-giecryal/documentos/ elaine-dos-santos-2020.pdf

32. Brasil. Portaria n. ${ }^{\circ} 1.156$, de 31 de agosto de 1990. Fica instituído, no Ministério da Saúde, o Sistema de Vigilância Alimentar e Nutricional SISVAN [serial on the internet]. Diário Oficial da União, Brasília (1990 Aug 31 [cited 2020 0ct 26]; Sec 1. Available from: http://189.28.128.100/ nutricao/docs/legislacao/portaria sisvan.pdf

33. Brasil. Nota técnica n. ${ }^{\circ}$ 11/2020-CGPROFI/ DEPROS/SAPS/MS [serial on the internet]. Brasillia (DF): Ministério da Saúde; 2020 [cited 2020 0ct 30]. Available from: https://bfa.saude.gov .br/public/file/1 2020 pandemia Covid-19.pdf

34. Mundim OS, Vidigal R, Michelotti FC, Pinto AJP. 0 Programa Bolsa Família e seus beneficiários na opinião pública brasileira. Opin. Publica [serial on the internet]. 2019 [cited 2020 Nov 8];25(3):556-76. Available from: http:// www.scielo.br/scielo.php?script $=$ sci arttext\&pid $=$ S0104-62762019000300556\&lng =pt\&nrm =iso

35. Brasil. Manual de condutas gerais do programa nacional de suplementação de vitamina A. Brasília (DF): Ministério da Saúde; 2013.

36. Brasil. Programa nacional de suplementação de ferro: manual de condutas gerais. Brasília (DF): Ministério da Saúde; 2013.

37. Brasil. Saúde da criança: aleitamento materno e alimentação complementar. 2. ed. Brasília (DF): Ministério da Saúde; 2015. 